Bibliotecas. Vol 36, № 2, julio-diciembre, 2018. EISSN: 1659-3286

URL: http://www.revistas.una.ac.cr/index.php/bibliotecas/index

DOI: http://dx.doi.org/10.15359/rb.36-2.3

Licencia: Creative Commons (BY-NC-SA) 4.0 Internacional

\title{
La voz del usuario en la planeación estratégica de bibliotecas públicas usando el Despliegue de la Función de la Calidad (QFD)
}

Users' Voice for Public Libraries Strategic Planning Using Quality Function Deployment (QFD)

\author{
José Refugio Romo-González \\ Universidad Autónoma de Chihuahua, México \\ ORCID: http://orcid.org/0000-0002-4810-4357 \\ Javier Tarango \\ Universidad Autónoma de Chihuahua, México \\ ORCID: http://orcid.org/0000-0002-0416-3400 \\ Juan D. Machin-Mastromatteo \\ Universidad Autónoma de Chihuahua, México \\ ORCID: http://orcid.org/0000-0003-4884-0474
}

Recibido: 27 de abril de 2018

Aceptado: 03 de mayo de 2018

Publicado: 06 de diciembre de 2018

\section{Resumen}

La planeación estratégica ha funcionado tradicionalmente al escuchar solo la voz de directivos. Además del estudio de sus ambientes y procesos funcionales, esta iniciativa considera como fundamental escuchar la voz del usuario, al valorar que son los que verdaderamente enfrentan las condiciones de la calidad de los servicios bibliotecarios. Por esta razón, se toman como referencia los resultados de un estudio de usuarios, en donde se aplicó la herramienta de Despliegue de la Función de la Calidad (QFD) en bibliotecas públicas del municipio de Chihuahua, México. Ello permitió identificar que la planeación estratégica de bibliotecas en un 
sistema integral administrativo puede estar sustentada en la gestión de la calidad y en el cuadro de indicadores de desempeño, cuya implantación podrá ser desarrollada bajo un ciclo de diseño, control, mejora e innovación para alcanzar objetivos armónicos y sistemáticos.

Palabras Clave: Usuarios de la información; Planeación Estratégica; Bibliotecas Públicas; Despliegue de la Función de la Calidad (QFD); Evaluación bibliotecaria; Servicios bibliotecarios; Calidad de los servicios.

\begin{abstract}
Given that strategic planning has traditionally worked by listening only to the voice of managers, in addition to studying their environments and functional processes, this initiative considers it fundamental to listen to the voice of the user, considering that they are the ones who truly face the conditions of the quality of library services. Therefore, the results of a user study are taken as a reference, where the Quality Function Deployment (QFD) tool was applied in public libraries in the municipality of Chihuahua, Mexico, which allowed identifying that the planning Strategic of libraries in an integral administrative system, can be sustained in the management of the quality and in the table of indicators of performance, whose implantation could be developed under a cycle of design, control, improvement and innovation to reach harmonic and systematic objectives.
\end{abstract}

Keywords: Information users; Strategic Planning; Public libraries; Deployment of the Quality Function Deployment (QFD); Library evaluation; Library services; Quality in services.

\title{
I. Introducción
}

La planeación estratégica no pronostica el futuro, pero a cualquier organización le puede ayudar a construirlo y además enfrentar con efectividad las contingencias (Fuentes, 2001; David, 2003). Las bibliotecas, como organismos clave en la prestación de servicios de información y como difusoras del conocimiento, no tienen por qué escapar a la premisa anterior y deben realizar en tiempos regulares, pero sobre todo en tiempos de crisis, análisis de tipo estratégico que les 
permita establecer de una manera sistemática y racional sus principales directrices, tales como su misión, visión, objetivos y estrategias institucionales (Fuentes Romero, 2002; Omella Clapalols, Permaniel Bastardad y Vilagrosa Alquézar, 2009; Buck, 2016; Dabbour y Kott, 2017).

El contenido del presente trabajo se desarrolla en tres partes, en la primera de ellas se propone realizar la planeación estratégica de las bibliotecas dentro de un sistema integral administrativo, cuya característica principal es la integración del modelo de planeación estratégica de David (2003), con el cuadro de mando integral de Kaplan y Norton (1996) y el modelo de gestión de la calidad de la Fundación Europea para la Administración de la Calidad - EFQM (Borbely, 2011; Robledillo y Velázquez, 2013; Vonhof y Bertele, 2017), el cual permitiría abordar de una manera holística el proceso de planeación estratégica y no como un proceso aislado, como tradicionalmente se trabaja a pesar de su trascendencia.

En la segunda parte del trabajo se resalta la importancia de escuchar la voz del usuario en la planeación estratégica de las bibliotecas, ya que en última instancia son los usuarios quienes disfrutan o sufren la calidad de los servicios bibliotecarios y obviamente tienen mucho que decir en sus correspondientes estrategias de desarrollo, cuya orientación prioritaria hacia los usuarios puede ser fuente no solo de ventajas competitivas, sino incluso de sobrevivencia institucional. Se presenta, asimismo, la herramienta Despliegue de la Función de la Calidad (QFD) como un instrumento ideal para escuchar y traducir la voz del usuario en estrategias de desarrollo de las bibliotecas (Chin, Pun, Leung y Lau, 2001).

En la última parte del trabajo, se presenta la descripción del proceso de aplicación de la QFD y sus resultados en la generación de estrategias de desarrollo para el Sistema de Bibliotecas Públicas de Chihuahua (municipio) México, a partir de un estudio estadístico de usuarios basado en una encuesta, en donde es posible observar la bondad de la QFD para traducir la voz del usuario en directrices estratégicas, que, a su vez, lo beneficiarán cuando estas se implementen. Finalmente se concluye, tanto sobre la metodología en sí como sobre la experiencia resultante de su aplicación. 


\section{La planeación estratégica de las bibliotecas dentro de un sistema integral administrativo}

Las bibliotecas, como la mayoría de las organizaciones, están sometidas a diversos procesos administrativos entre los que destacan los análisis estratégicos para definir su misión, visión, objetivos y principales estrategias (Kundanis, 2014), pero también resaltan los procesos de gestión de la calidad, procesos de acreditación o certificación mediante el cumplimiento de ciertos estándares en los indicadores de desempeño bibliotecario (Aponte y Chávez, 2008; Melo y Zavala, 2012). Sin embargo, la mayoría de estos procesos se realizan de una manera aislada e incluso limitándose al diagnóstico (Dabbour y Kott, 2017); por ende, sus resultados no siempre son los deseados 0 , en el mejor de los casos, se realizan con poca información acerca de otros procesos administrativos relacionados, lo cual redunda en una escasa o baja alineación de dichos procesos con la visión integral de la institución (Friss, 2003; Ministerio de Sanidad y Consumo, 2007). Es decir, la gestión estratégica, la gestión de la calidad, el manejo de los indicadores de desempeño, etc., se abordan como proyectos independientes, con escasa relación o, en su defecto, como un portafolio controlado de proyectos, cuando en realidad lo que se requiere es un enfoque de sistemas, que involucre a todos los subsistemas organizacionales e incluya un análisis de carácter holístico (Dabbour y Kott, 2017).

Por lo anterior, se propone la implantación de un sistema integral administrativo (SIAD) que se sustente en un modelo de calidad y que vaya ligado con subsistemas de planeación y medición del desempeño, incorporándolos y orientándolos de manera integral hacia el cumplimiento de la misión y visión de la biblioteca de que se trate (Gatti, 2008). La implantación de dicho sistema deberá hacerse bajo un ciclo de diseño, control, mejora e innovación (Ciclo DINOME) que permita alcanzar los objetivos institucionales de una manera armónica y sistematizada (UACH, 2011).

Realizar esfuerzos aislados o con falta de coordinación, aunque de buena voluntad, llevan inevitablemente al desperdicio de recursos y a la falta de eficacia organizacional. Un sistema basado en un modelo de calidad, aunque sencillo, pero claro y preciso, puede ser la diferencia. En consecuencia, se proponen usar tres modelos básicos, el primero y más importante es un 
modelo que sirva para gestionar la calidad de los servicios que presta la biblioteca; el segundo para la administración de las estrategias bibliotecarias marcadas en los planes de desarrollo de la institución que albergue a dicha instancia y el tercero para evaluar el desempeño organizacional de la misma biblioteca. Los tres modelos se presentan interactuando de manera coordinada dentro del Sistema Integral Administrativo (SIAD) de la biblioteca y permitiría vigilar la marcha adecuada de la calidad, las estrategias y los indicadores del desempeño de la biblioteca.

El modelo de gestión de la calidad de la Fundación Europea para la Administración de la Calidad (EFQM) es un modelo propuesto en 1991 y se utiliza tanto para implantar sistemas de calidad, como para su gestión y evaluación. Actualmente, es utilizado por la Secretaría de Educación Pública de México (Moreno Alego, 2007; SEP, 2005) para enfocar el sistema educativo nacional hacia la calidad e innovación. Además, es un modelo que también ha sido adoptado por diversas instituciones de educación superior en México y desde luego europeas.

El modelo de calidad EFQM enfatiza una idea central: la necesidad de orientar todos los esfuerzos y tareas a lograr resultados que generen valor a quienes han sido identificados como "clientes" tanto internos como externos: estudiantes, padres de familia, empleadores, trabajadores académicos y administrativos, autoridades gubernamentales y sociedad en general, tal como lo muestra la Figura 1.

Por otro lado, la planeación es un proceso continuo y no un evento (Pacios, 2017). Las estrategias se derivan de la misión y la visión organizacional (Kundanis, 2014). Sin embargo, sin una buena implementación y un buen control de las estrategias, cualquier misión y visión serán inalcanzables o serán simples buenos deseos. Para el caso de la administración de estrategias, se propone aplicar el modelo de Fred David (2003), que es un modelo que sigue las directrices básicas de la Administración Estratégica y el cual se divide en tres fases: (i) formular estrategias; (ii) implementar estrategias; y (iii) evaluar estrategias, representado en la Figura 2. 
Figura 1

Modelo de gestión de calidad de la EFQM

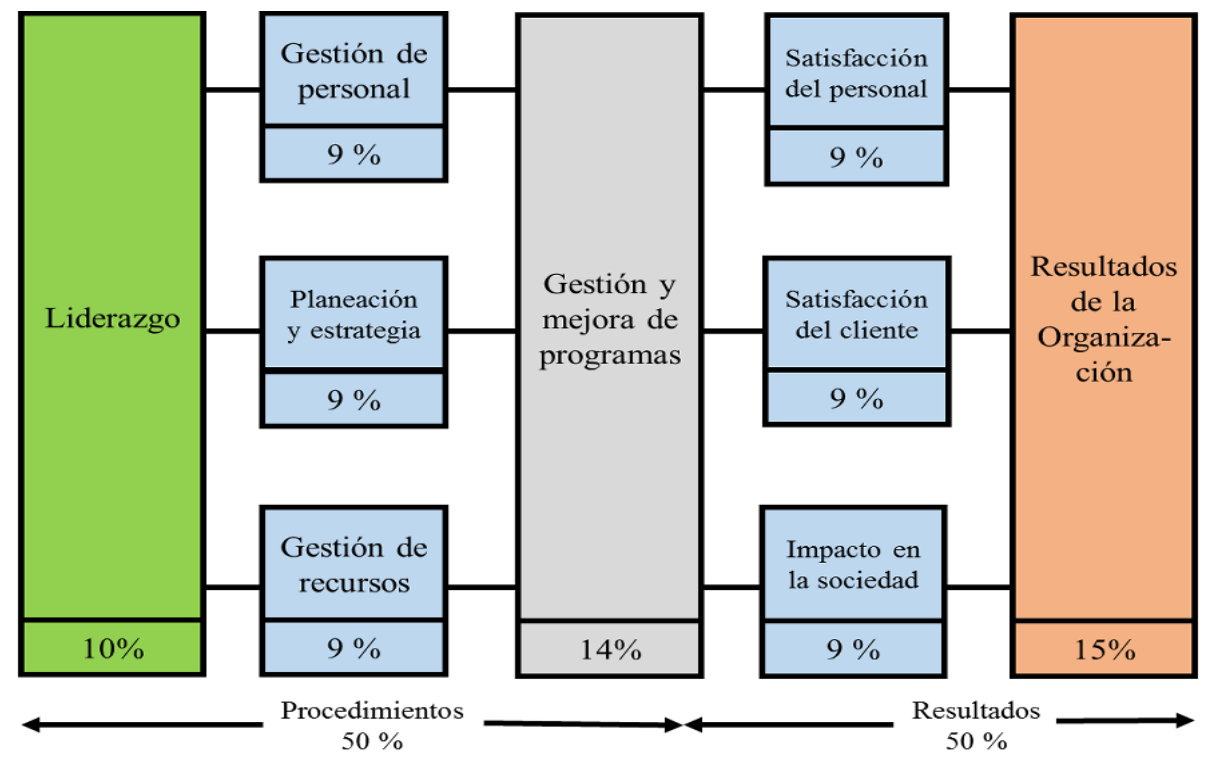

Fuente: Moreno, J. (2007). Guía para la aplicación del modelo EFQM en entidades de acción social.

En tercera instancia, para administrar el desempeño de una biblioteca, a través de un cuadro de mando integral con indicadores definidos por la institución, se propone aplicar el modelo de Kaplan y Norton (1996) o Balanced Score Card (BSC) el cual es un modelo que actualmente se está empleando como un Sistema de Administración del Desempeño (De Melo y De Alencar, 2016), que toma en cuenta no solo la perspectiva financiera/efectividad, sino que agrega otras tres perspectivas: clientes, personal y procesos. El modelo de mando integral de Kaplan y Norton (ver Figura 3) es un modelo para administrar el desempeño estratégico de las organizaciones. Por cada una de las 4 perspectivas planteadas para darle seguimiento al desempeño institucional, se definen cierto número de criterios e indicadores medibles, que posteriormente se transforman en un "tablero de mando" que obviamente le indica a la organización en cuáles áreas estratégicas se tiene un alto o bajo desempeño, permitiéndole a la biblioteca detectar a tiempo posibles desviaciones estratégicas y estar en condiciones de tomar acciones preventivas y/o correctivas. 
Figura 2

Modelo de administración estratégica de Fred David

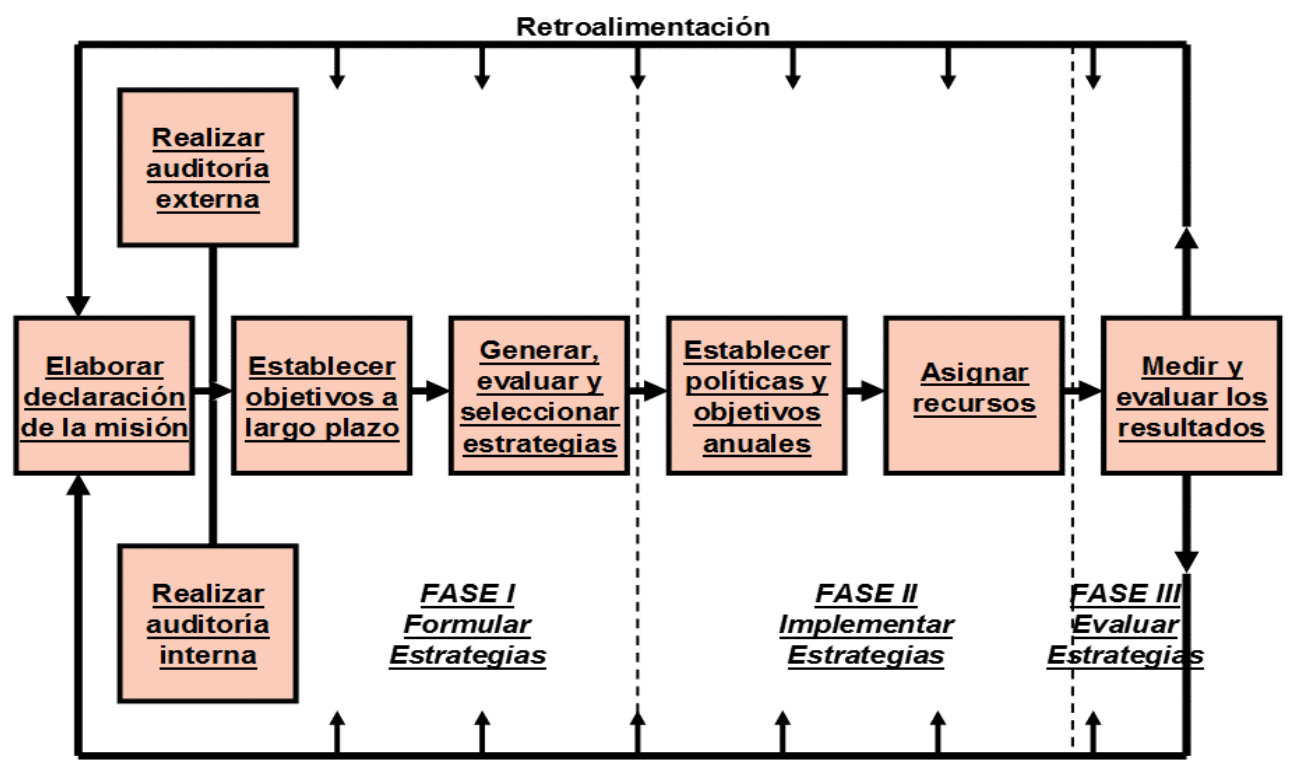

Fuente: David, F. (2003). Conceptos de administración estratégica.

Figura 3 Modelo de mando integral de Kaplan y Norton

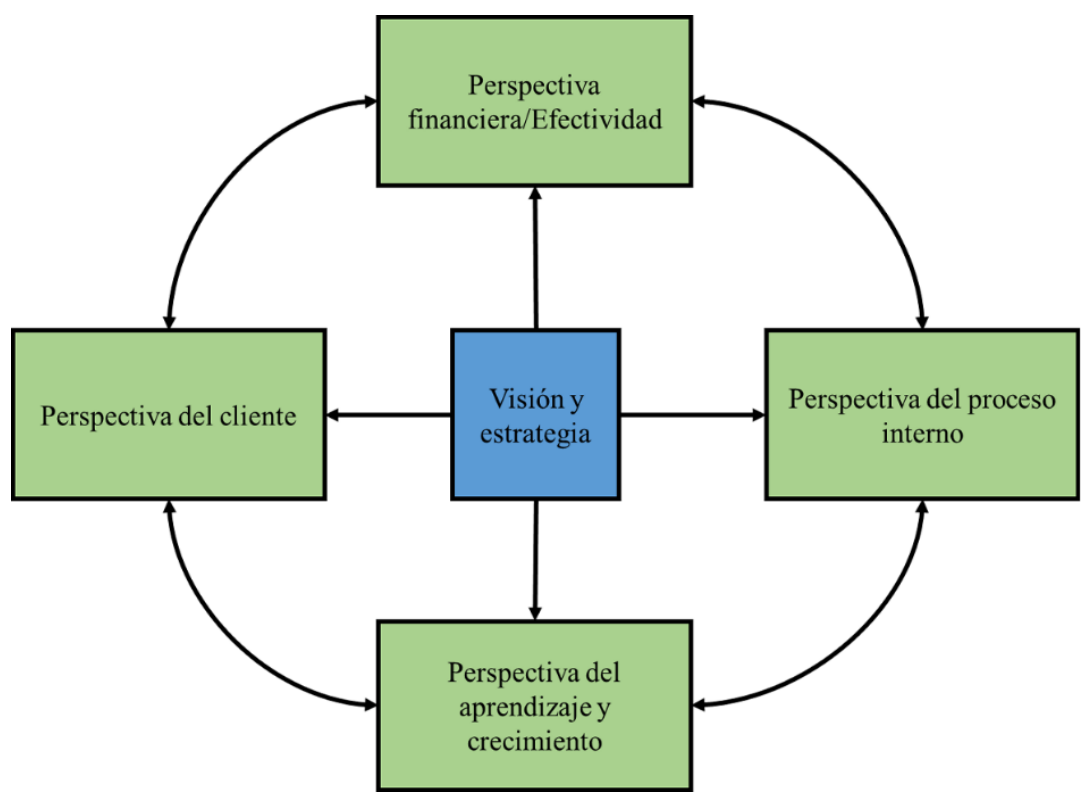

Fuente: Kaplan, R. y Norton, D. (1996). The balanced scorecard. 
En este trabajo se propone que los tres modelos anteriores se apliquen bajo un enfoque sistémico que integre en un solo sistema administrativo lo más sobresaliente de cada uno de los tres modelos. El basamento del Sistema Integral Administrativo (SIAD) es el modelo de gestión de la calidad, mismo que deberá marcar los subsistemas organizacionales, procesos e indicadores principales para que se pueda implantar una filosofía y gestión de la calidad en la biblioteca que redunde en la prestación de servicios informativos con un alto nivel de excelencia.

Aunque cada modelo tiene sus fronteras propias, es posible su integración formal, ya que cada uno de ellos fue ideado originalmente para identificar la eficiencia su función, pero sin que ninguno de sus autores lo hubiera ideado para trabajar aisladamente. El Sistema Integral Administrativo que se propone para la biblioteca se representa en la Figura 4. Sistema integral administrativo: gestión de calidad-estrategias-desempeño.

\section{Figura 4}

\section{Sistema integral administrativo: gestión de calidad-estrategias-desempeño}

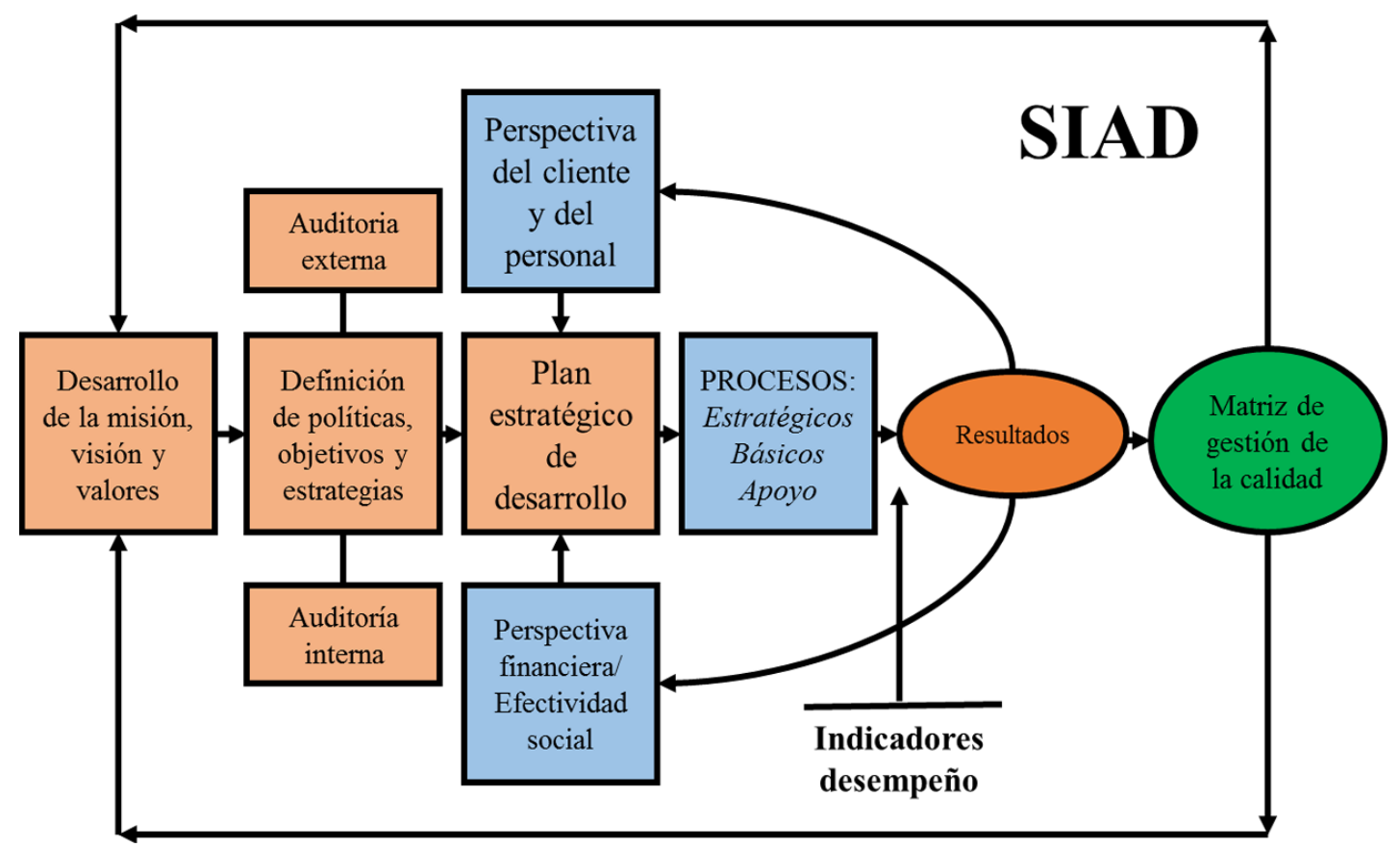

Fuente: Elaboración propia con referencia en los modelos EFQM, David y Kaplan-Norton 
Para implantar el SIAD se requiere aplicar un ciclo de diseño, innovación y mejora continua (Ciclo DINOME) que permita tanto el diseño de subsistemas como de sus procesos asociados; la definición de los indicadores de desempeño bibliotecario que pueden ser provenientes de la Dirección de la organización y de la propia biblioteca, así como de organismos externos acreditadores y/o certificadores de bibliotecas; la implantación de sistemas, procesos e indicadores; la medición del desempeño; el análisis, seguimiento, diagnósticos y auditorías; identificación de brechas; comparación con las mejores prácticas; adecuación, medidas preventivas y correctivas; y subciclos de mejora continua, innovación y comparación referencial. Todo ello cerrando con la mejora e innovación en la biblioteca, que volvería a plantearse el inicio del ciclo con el rediseño de los subsistemas y procesos. En diagrama de flujo (representado en la Figura 5) muestra con claridad el Ciclo DINOME que se propone para que una biblioteca pueda implementar su sistema integral administrativo SIAD, basado fundamentalmente en un modelo de gestión de la calidad.

Figura 5

Ciclo de diseño, innovación y mejora para implementar el Sistema Integral Administrativo (SIAD)

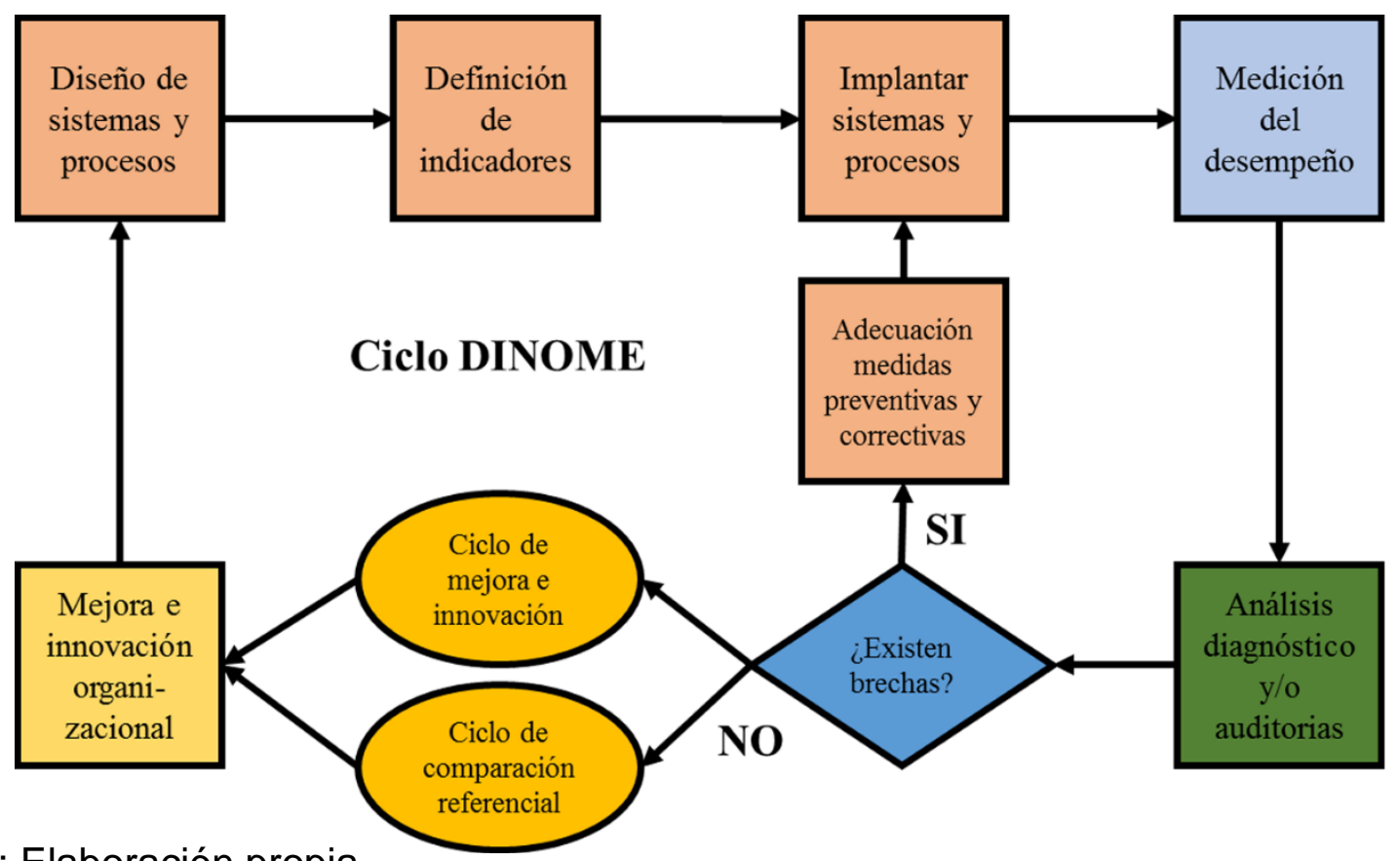

Fuente: Elaboración propia. 


\section{La voz del usuario y la QFD}

Las bibliotecas como cualquier otra organización deben encaminar sus esfuerzos a satisfacer las necesidades y requerimientos de los usuarios (Boyce, 2017), ya que estos son su razón de ser. Es el usuario quien califica en última instancia, la calidad de los servicios bibliotecarios y quien define el verdadero valor de estos (Madrid, 2002).

Una organización no puede generar usuarios leales sin crear primero usuarios satisfechos y no puede satisfacer a sus usuarios sin antes conocerlos y escucharlos (Evans y Lindsay, 2005), por lo que resulta trascendente idear mecanismos o herramientas que permitan a las bibliotecas "escuchar la voz del usuario" (Bawden, Petuchovaite y Vilar 2005), no solo en aspectos operativos o relacionados con la mejora de procesos, servicios o productos, sino incluso en cuestiones estratégicas.

Los usuarios de las bibliotecas perciben los servicios que se les brindan, desde el punto de vista de los beneficios que estos les proveen y los problemas que les resuelven. En consecuencia, su perspectiva difícilmente se alinea con los procesos singulares de la biblioteca y su visión permea o atraviesa los diferentes procesos, personal bibliotecario, servicios y políticas de operación de esta, es decir el usuario visualiza factores como la calidad, confiabilidad, empatía, seguridad, etc. (Blázquez y Medino, 2005); mientras que la visión de los directivos de la biblioteca se focaliza en factores tales como el personal, los recursos financieros, las operaciones y los servicios, por lo cual sería pertinente hacer la pregunta ¿se tiene una verdadera "visión del usuario" o se está cayendo en la trampa de usar la "visión de la organización" para dirigir y operar la biblioteca?

Usualmente la voz preponderante en los ejercicios de planeación estratégica de las bibliotecas es la voz de los directivos, con su muy particular forma de ver las cosas, aunque también se escucha, pero en menor grado a la voz del personal operativo en ciertos momentos del análisis estratégico, además de la voz de los procesos y desde luego la voz de los agentes externos (patrocinadores, sindicatos, etc.), dando origen con ello a las estrategias directivas, estrategias consultadas, estrategias implícitas y a las estrategias externas, respectivamente (González, 
2012). Sin embargo, la voz del usuario se escucha muy tenuemente o incluso no se le toma en cuenta en los análisis estratégicos a pesar de que en última instancia son los usuarios quienes disfrutan o sufren la calidad de los servicios bibliotecarios y obviamente tienen mucho que decir en sus correspondientes estrategias de desarrollo, donde una orientación prioritaria hacia los usuarios puede ser fuente no solo de ventajas competitivas, sino incluso de sobrevivencia institucional (Martín-Montalvo, 2007).

No se trata de minimizar la voz institucional, sino de darle su debido lugar e importancia a la voz de los usuarios y armonizarla con la visión de los directivos y también con las voces de los demás actores que también tienen algo que decir en la administración estratégica de las bibliotecas. ¿Cuántas organizaciones que han fracasado, argumentan su declive por no haber escuchado a tiempo la voz de sus usuarios? Seguramente una proporción muy alta. En consecuencia, es tiempo de que las bibliotecas salgan de su encapsulamiento y aborden a sus usuarios interrogándolos acerca de sus necesidades y requerimientos, y de las cuestiones estratégicas que gobiernan la vida institucional de la biblioteca misma. Sin embargo "escuchar la voz del usuario" de las bibliotecas entraña muchas dificultades, tales como:

a) La expresión de las necesidades de los usuarios requiere de una "traducción técnica".

b) Los usuarios hablan en "muchos idiomas".

c) En las manifestaciones de los usuarios existen a veces "mensajes ocultos".

d) Algunas necesidades no son identificadas ni por los mismos usuarios.

e) Los servicios bibliotecarios pueden presentar "usos ocultos".

f) En ocasiones el usuario requerirá del acompañamiento de personal bibliotecario para registrar el verdadero uso de los servicios que se le brindan.

g) La voz del usuario debe ser escuchada dimensionando problemas, es decir escuchando quejas, y también en términos de aprovechamiento de oportunidades.

Por lo anterior, es obvio el requerimiento de técnicas instrumentales o herramientas que permitan "escuchar la voz del usuario" y traducirla en términos estratégicos u operativos para la biblioteca. Evans y Lindsay (2005) consignan dos herramientas usadas frecuentemente en el 
área de gestión de la calidad para "escuchar la voz del usuario": el Despliegue de la Función de Calidad (DFC) o Quality Function Deployment (QFD) y la Ingeniería de Conceptos (IC), siendo la QFD la herramienta más conocida y desarrollada (Gutiérrez, Gutiérrez, Garibay y Díaz, 2014).

En este trabajo se propone el uso de la QFD para traducir la voz del usuario en aspectos estratégicos del desarrollo de la biblioteca y no solo emplearla en el diseño y mejora operativa de los servicios bibliotecarios, como tradicionalmente se ha venido usando, con lo cual se ampliaría y enriquecería su aplicación y utilidad. La QFD fue originalmente ideada en 1966 como un método para desarrollar la calidad en el diseño de productos y servicios y encaminada primordialmente a satisfacer las necesidades de los clientes (Hunt y Xavier, 2003), aunque posteriormente fue adoptada por empresas como Toyota Motors y muchas otras.

La Asociación Latinoamericana de QFD (www.qfdlat.com) considera que el QFD es un sistema que busca focalizar el diseño de los productos y servicios en dar respuesta a las necesidades de los clientes. Esto significa alinear lo que el cliente requiere con lo que la organización produce, permitiéndole a una organización entender la prioridad de las necesidades de sus clientes y encontrar respuestas innovadoras a esas necesidades, a través de la mejora continua de los productos y servicios en búsqueda de maximizar la oferta de valor (Tamayo y González, 2009), por lo tanto, a través del QFD todo el personal de una organización puede entender lo que es realmente importante para los clientes y trabajar para cumplirlo.

Para 1980 la QFD atrae la atención de los expertos occidentales de la calidad y pronto se descubre el poder adicional de las técnicas y matrices de la QFD en la planeación estratégica, hoy en día existen diversas aplicaciones documentadas de la QFD en aspectos estratégicos de todo tipo de empresas e instituciones. De hecho, Hunt y Xavier (2003), quienes han investigado la aplicación de la QFD en la planeación estratégica de las organizaciones, señalan que el poder de esta herramienta radica en que la QFD ayuda a identificar qué es importante, al proveer un sistema lógico para reemplazar la toma de decisiones basada en emociones. Es decir, aunque la QFD nace y se desarrolla originalmente para el diseño de productos y servicios, ha encontrado posteriormente utilidad en la planeación estratégica de las organizaciones y 
actualmente se le usa adicionalmente en campos tan diversos como: análisis de segmentación de mercados, diseño Six-Sigma, selección de conceptos, optimización en la asignación de recursos, desarrollo de productos estrella, diseños focalizados en los clientes, análisis de modos y efectos de fallas (FMEA), diseños compensatorios, etc., tales ideas son recurrentes en las propuestas de Herrera-Viedma, López y Vílchez, 2006; García, 2009; y Varela, 2012.

La QFD utiliza una serie de matrices para relacionar la voz del usuario con las características técnicas de un servicio, los requerimientos de sus componentes, la planeación para el control de procesos y las operaciones de prestación de los servicios, desarrollando a través de cuatro fases o etapas. La integración de las diversas matrices en la primera fase genera lo que se conoce como la "casa de la calidad", representada en la Figura 6 (QFD).

\section{Figura 6}

\section{Casa de la calidad}

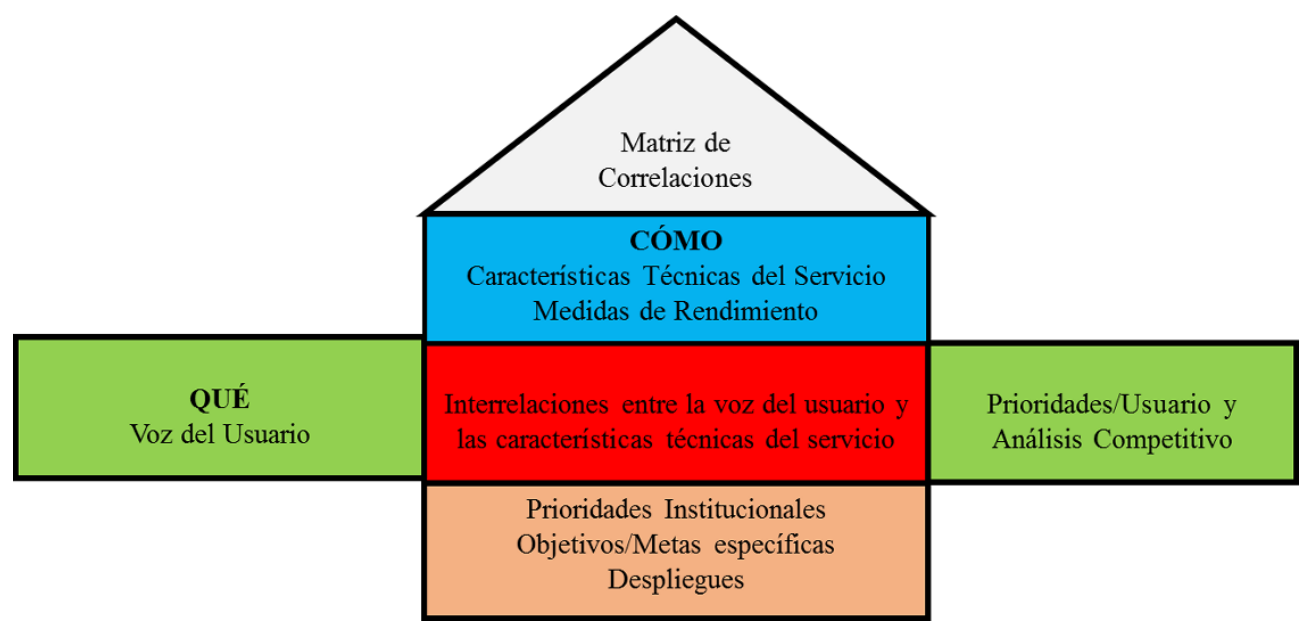

Fuente: Elaboración propia tomando como referencia los elementos de QFD.

La "casa de la calidad" para una QFD aplicada a planeación estratégica, sería básicamente similar a la anterior, solo que, en lugar de las características técnicas del servicio en la matriz de los Cómo, se tendrían las estrategias organizacionales de desarrollo. Hunt y Xavier (2003), proporcionan un análisis pormenorizado de las variantes autorales acerca de la QFD aplicada a cuestiones estratégicas, además de comparar esta herramienta con la técnica "Hoshin Kanri" 
para el despliegue de políticas estratégicas y proponer un proceso integral de análisis estratégico que partiendo de la misión, se escuche tanto la voz de los clientes, como la de los directivos y otros grupos de influencia en la organización, así como de los procesos productivos, al cual le llaman "Proceso $C D-M A P$ ".

Los pasos básicos para construir la tradicional "casa de la calidad", según Evans y Lindsay (2005) serían:

a) Identificación de las necesidades del usuario.

b) Traducción de las necesidades del usuario en requerimientos técnicos del servicio.

c) Relacionar las necesidades del usuario con los requerimientos técnicos.

d) Priorización de las necesidades del usuario y análisis competitivo de los servicios.

e) Análisis de prioridades institucionales y establecimiento de objetivos específicos.

f) Establecer cuáles requisitos técnicos del servicio se deben desplegar en el resto del proceso.

Estos mismos pasos, con las debidas adecuaciones, se aplicarían en un análisis QFD específico para la planeación estratégica. Cabe resaltar que en la actualidad se ofrecen en el mercado programas de cómputo que facilitan la aplicación de la QFD en diversos campos, desde el diseño de productos hasta la planeación estratégica y varios más. El abanico de programas computacionales va desde los más sencillos como, por ejemplo, el SmartDraw que proporciona la plantilla gráfica para vaciar las matrices de la casa de la calidad, hasta los más completos como el QFD Designer que incluye la mayoría de los análisis que requiere el QFD con sus respectivas variantes según el campo de aplicación que elija el usuario.

\section{Objetivo del estudio}

Establecer un procedimiento de planeación estratégica en bibliotecas públicas de Chihuahua (municipio), México, dentro de un sistema integral administrativo, cuya característica principal es la integración de diversos modelos administrativos que permitan abordar, de manera holística, el proceso de identificación de elementos para la mejora a partir de la voz de los 
usuarios, de tal forma que se permita alcanzar los objetivos bibliotecarios de forma armónica y sistemática.

\section{Aplicación del QFD en el desarrollo de estrategias: caso bibliotecas públicas de Chihuahua (municipio), México}

A continuación, se presenta la descripción del proceso de aplicación de la QFD y sus resultados en la generación de estrategias de desarrollo para las bibliotecas públicas de Chihuahua (municipio), México, a partir de un estudio estadístico de usuarios basado en una encuesta. La red de bibliotecas públicas estatales estudiadas fueron nueve entidades: Biblioteca Pública Central Estatal (CIDECH), Biblioteca Pública "Colinas del Sol”, Mediateca Municipal, Biblioteca Pública ISSSTE-CNCA No. 1, Biblioteca Municipal, Centro Cultural Bicentenario "Carlos Montemayor", Biblioteca Fundadores, Biblioteca del H. Congreso del Estado de Chihuahua e ICHICULT, todas ubicadas en zonas urbanas.

El análisis de la "voz del usuario" a través de la QFD, se sustentó en datos empíricos provenientes de un estudio de usuarios para la mencionada red de bibliotecas, realizado en el municipio de Chihuahua, México, durante el primer semestre de 2016 considerando una muestra probabilística para el caso de proporciones y poblaciones infinitas (mayores a 10,000 sujetos), con una probabilidad de ocurrencia del 50\% para la variable de satisfacción con el servicio de la biblioteca y por contraparte un $50 \%$ de usuarios no satisfechos; empleando un nivel de confianza del 95\% y un margen de error del 5\%, lo que arrojó un tamaño de muestra de 384 usuarios como mínimo estadístico, el cual fue cubierto satisfactoriamente al obtener 400 casos útiles, los cuales fueron capturados y analizados en el programa estadístico SPSS versión 20. El Cuadro 1 consigna la ficha técnica muestral correspondiente a esta investigación, la cual incluye los datos poblacionales y muestrales para el período en estudio, así como los parámetros de diseño empleados. 


\section{Cuadro 1}

Ficha técnica muestral: usuarios de bibliotecas públicas del municipio de Chihuahua

\begin{tabular}{ll}
\hline \multicolumn{1}{c}{ Aspectos clave } & \multicolumn{1}{c}{ Parámetros } \\
\hline Población & $\begin{array}{l}\text { Usuarios de bibliotecas } \\
\text { públicas de Chihuahua } \\
\text { Un usuario }\end{array}$ \\
Unidad de análisis & $\begin{array}{l}\text { Infinito (mayor a 10,000) } \\
\text { Tamaño (N) }\end{array}$ \\
Criterios de inclusión & Acude a un servicio \\
Criterios de exclusión & Ninguno \\
\hline Muestra & \\
Nivel de confianza & $95 \%$ \\
Probabilidad ocurrencia / no & $50 \%$ / 50\% \\
ocurrencia & satisfechos/insatisfechos \\
Margen de error de diseño & $5.0 \%$ \\
Tamaño de diseño & 384 mínimo estadístico \\
Tamaño final (n) & 400 casos útiles \\
Margen de error final & $4.91 \%$ \\
\hline Marco muestral & Servicios bibliotecarios \\
\hline Muestreo & \\
Método de muestreo & Aleatorio \\
Selección de unidades & Sistemática \\
\hline
\end{tabular}

Fuente: Elaboración propia.

El instrumento de medición cubrió cinco apartados: (i) uso de los servicios bibliotecarios; (ii) cobertura de las necesidades informativas de los usuarios; (iii) nivel de satisfacción con la calidad de los servicios prestados; (iv) inconformidades o quejas sobre los procesos de prestación de servicios; y (v) áreas de oportunidad para mejorarlos a través de sugerencias. 
Debido al carácter de la biblioteca, la muestra analizada incluyó diversos segmentos de edad tales como niños, adolescentes, jóvenes, adultos y adultos mayores; variadas ocupaciones, desde los estudiantes, sobre todo de preparatoria y secundaria, que ocuparon la mayor carga muestral (80\%), provenientes de más de 120 instituciones educativas, hasta personas económicamente activas (empleados y empresarios), amas de casa, jubilados, e incluso desempleados; la muestra también incluyó distintos niveles socioeconómicos, aunque la más numerosa fue la clase media baja y media; también se incluyó en el estudio una cantidad de colonias urbanas cercana a la centena.

El análisis de los instrumentos utilizados mostraron, con respecto a los resultados de uso y cobertura de los servicios bibliotecarios, que el 39\% de los entrevistados se clasificaron como usuarios frecuentes (visitas semanales o al menos dos o tres veces por mes), usando con mayor incidencia los servicios de consulta de material general y especializado (57\%) y en menor medida otros servicios como préstamos a domicilio, préstamos de revistas y periódicos, préstamo de material audiovisual, visitas guiadas, uso de equipo de cómputo, préstamos de diccionarios y enciclopedias, etc. Sin embargo, el 60\% manifestó desconocer toda la gama de servicios que ofertaba la biblioteca, aunque el $75 \%$ señaló encontrar con rapidez la información que buscaba al acudir a la biblioteca. Asimismo, el 92\% indicó que la biblioteca le proporciona actualmente los servicios requeridos, los cuales se prestan con oportunidad (86\%) y disponibilidad (85\%), siendo estos de utilidad y actualizados (83\%).

En relación con la opinión de los usuarios sobre la calidad de los servicios de información que presta la citada biblioteca, el $87 \%$ manifestó que la oferta de servicios corresponde a sus necesidades de información, mostrándose un $85 \%$ satisfecho con el trato en la atención que se brinda a los usuarios por parte del personal encargado de la institución. El índice general de satisfacción con la biblioteca arrojó un $86 \%$ de usuarios satisfechos.

El último apartado del instrumento de medición, referente a las inconformidades o quejas sobre los procesos de prestación de servicios y a las áreas de oportunidad para mejorarlos a través de sugerencias, es el apartado que en esencia se utiliza en este trabajo para realizar el análisis 
QFD y poder "escuchar la voz del usuario" para obtener directrices estratégicas que guíen la marcha de la biblioteca, no olvidando que apenas sería una de las voces que se deben escuchar, ya que los directivos y otros grupos de interés también deben escucharse, así como a la "voz" de los procesos mismos.

La recolección de datos en el último reactivo, que como se indica, forma la parte fundamental del análisis de este artículo, representó solicitarle a los encuestados, aquellos aspectos que favorecen o no favorecen el uso de los servicios bibliotecarios, tomándose como datos de análisis los aspectos no favorables, ya que se considera como aquellos verdaderamente sustanciales a la voz real de los usuarios, por lo que en general, se recolectaron datos en lenguaje natural, mismo que fue convertido, por cuestiones de manejo de datos objetivos, en lenguaje controlado. A partir de esto se procedió a la siguiente aplicación metodológica:

\section{a. Análisis QFD}

Como ya se mencionó anteriormente, aquí se permite dar entrada a la información recopilada y colocarla en una estructura llamada "la casa de la calidad", que lleva a su aprovechamiento, ya sea para diseñar o mejorar los servicios bibliotecarios o bien para obtener directrices estratégicas focalizadas en la atención del usuario, existiendo varias herramientas que se pueden aplicar de manera previa a la construcción de la "casa de la calidad", de las cuales Villardefrancos Álvarez (2004) menciona al menos cinco de ellas:

a) Tabla del Contexto del Usuario (TCU).

b) Tabla de Traducción del lenguaje del Usuario sobre inconformidades y sugerencias para mejoras e innovación (TTU).

c) Tabla de la Voz del Usuario (TVU).

d) Diagrama de afinidad o técnica TKJ.

e) Matriz de priorización.

Aunque desde luego no existe limitante alguna en cuanto al número y tipo de herramientas previas que puedan aplicarse para procesar o evaluar la información que se empleará en la casa de la calidad. Aquellas pertinentes a este estudio se describen a continuación. 


\section{b. Tabla de contexto (TCU)}

Esta tabla presenta las características de uso de los servicios bibliotecarios por parte de los usuarios, tal y como ellos lo manifiestan, pudiéndose segmentar por tipo de usuario. Es decir, caracteriza o contextualiza el ambiente bajo el cual el usuario hace uso de los servicios, respondiendo a las interrogantes clásicas sobre: ¿quién?, ¿para qué?, ¿por qué?, ¿dónde?, ¿cuándo? y ¿cómo? Enseguida se reporta únicamente la TCU para el usuario mayoritario $(80 \%$ de los usuarios), es decir estudiantes de secundaria y preparatoria, de la biblioteca bajo estudio (Cuadro 2)

\section{Cuadro 2}

Contexto del usuario (TCU)

\begin{tabular}{|c|c|c|c|c|c|}
\hline ¿Quién? & ¿Para qué? & ¿Por qué? & ¿Dónde? & ¿Cuándo? & ¿Cómo? \\
\hline - Usuario de & -Hacer tareas & - Obligación & - Bibliotecas & -De lunes a & -Solicitando \\
biblioteca & escolares & escolar & públicas del & viernes de & servicios de \\
(generalmente & -Desarrollar & - Superación & municipio de & 8 am a 8 & biblioteca \\
estudiante de & proyectos & personal & Chihuahua & pm y & \\
nivel & -Indagaciones & - Entreteni- & & sábados de & \\
secundario o & temáticas & miento & & 9 am a 2 & \\
preparatoria) & & & & pm & \\
\hline
\end{tabular}

Fuente: Elaboración propia.

\section{c. Cuadro de Traducción del Lenguaje del Usuario sobre Inconformidades y Mejoras (TTU)}

Se escucharon un total de 92 inconformidades o quejas sobre los procesos de prestación de servicios de la biblioteca, aplicándose un análisis de afinidad sobre las mismas para categorizarlas y un análisis frecuencial para posteriormente establecer su importancia relativa. Asimismo, los usuarios encuestados manifestaron 116 sugerencias o recomendaciones para mejorar los procesos de servicios bibliotecarios, detectándose por lo tanto diversas áreas de oportunidad de mejora e innovación y al igual que en el caso anterior, se aplicó un análisis de afinidad sobre dichas recomendaciones para categorizarlas y un análisis frecuencial para 
posteriormente establecer su importancia relativa. El Cuadro 3 reporta la TTU, reducida por razones de ejemplificación, tanto para inconformidades como para mejoras e innovación.

\section{Cuadro 3}

Traducción del lenguaje del usuario sobre inconformidades y mejoras (TTU)

\begin{tabular}{|c|c|c|}
\hline Tipo & Expresiones del Usuario & $\begin{array}{c}\text { Traducción del Lenguaje del } \\
\text { Usuario }\end{array}$ \\
\hline Quejas & $\begin{array}{l}\text { - Son muy estrictos en los requisitos. } \\
\text { - Hay discriminación por parte de los } \\
\text { bibliotecarios. } \\
\text { - A veces no está el encargado. }\end{array}$ & $\begin{array}{l}\text { - Trámites burocratizados para } \\
\text { solicitar y obtener servicios. } \\
\text { - Trato descortés e indiferente por } \\
\text { parte del personal de la biblioteca. } \\
\text { - Ausencias temporales en los puestos } \\
\text { de trabajo por parte del personal. }\end{array}$ \\
\hline Mejora & $\begin{array}{l}\text { - Un método más rápido de consulta. } \\
\text { - Más atención. }\end{array}$ & $\begin{array}{l}\text { - Mejorar o implantar sistemas más } \\
\text { eficientes para realizar consultas. } \\
\text { - Incrementar el número de personal } \\
\text { que presta directamente los servicios. }\end{array}$ \\
\hline Innovación & $\begin{array}{l}\text { - Libros de medicina. } \\
\text { - Que la hagan un poco más } \\
\text { tecnológica. }\end{array}$ & $\begin{array}{l}\text { - Introducir material sobre literatura } \\
\text { especializada (Ciencias de la Salud). } \\
\text { - Implantar nuevas tecnologías } \\
\text { (automatización, TIC, etc.) }\end{array}$ \\
\hline
\end{tabular}

Fuente: Elaboración propia.

\section{d. Cuadro de la Voz del Usuario (TVU)}

Esta tabla permite establecer la calidad demandada por la voz del usuario una vez ya traducida, sobre distintos factores de la operativa organizacional, especificando los atributos de calidad correspondientes tales como funciones, tiempos, costos, etc., que posteriormente serán datos útiles para la elaboración de la casa de la calidad. En el Cuadro 4 se presenta la TVU, también reducida con fines de ejemplificación. 


\section{Cuadro 4}

\section{La voz del usuario (TVU)}

\begin{tabular}{|c|c|c|}
\hline $\begin{array}{c}\text { Factor } \\
\text { Organizacional }\end{array}$ & $\begin{array}{c}\text { Calidad } \\
\text { Demandada }\end{array}$ & $\begin{array}{c}\text { Atributos de } \\
\text { Calidad }\end{array}$ \\
\hline $\begin{array}{c}\text { Personal de la } \\
\text { biblioteca }\end{array}$ & $\begin{array}{l}\text { - Amigable y cortés. } \\
\text { - Capacitado en su área. } \\
\text { - Disponible cuando se le } \\
\text { requiere. }\end{array}$ & $\begin{array}{l}\text { - Motivación (cursos e incentivos). } \\
\text { - Entrenamiento, capacitación } \\
\text { profesionalización. } \\
\text { - Número de empleados y horarios de } \\
\text { trabajo. }\end{array}$ \\
\hline Instalaciones & $\begin{array}{l}\text { - Mayor amplitud. } \\
\text { - Funcionalidad. } \\
\text { - Mejor ambiente físico. }\end{array}$ & $\begin{array}{l}\text { - Espacios adecuados }\left(\mathrm{m}^{2}\right) \text {. } \\
\text { - Comodidad para estudiar, hacer } \\
\text { tareas y trabajar en equipo. } \\
\text { - Iluminación, nivel de ruido, ventilación } \\
\text { temperatura. }\end{array}$ \\
\hline
\end{tabular}

Fuente: Elaboración propia.

Enseguida se determina la importancia relativa que los usuarios otorgan a cada uno de los aspectos de la calidad demandada, pudiéndose calificar las prioridades del usuario mediante ponderaciones en una escala del 1 al 5 , siendo el 5 el aspecto más importante para el usuario y el 1 el aspecto menos importante.

Para la biblioteca que ocupa este caso, la ponderación se obtuvo por medio de un análisis frecuencial de las 92 inconformidades y las 116 recomendaciones ya categorizadas, de manera que los niveles más altos de frecuencia se les asignó un factor de ponderación de 5 puntos y los de más baja frecuencia un factor de ponderación de 1 punto. Finalmente, integrando toda la información anterior y sus calificaciones respectivas se obtuvo una matriz de calidad o "casa de la calidad" (ver figura 7). 
Bibliotecas. Vol. 36, № 2, julio-diciembre, 2018. EISSN: 1659-3286

URL: http://www.revistas.una.ac.cr/index.php/bibliotecas/index

Figura 7

Resultados de la matriz de calidad

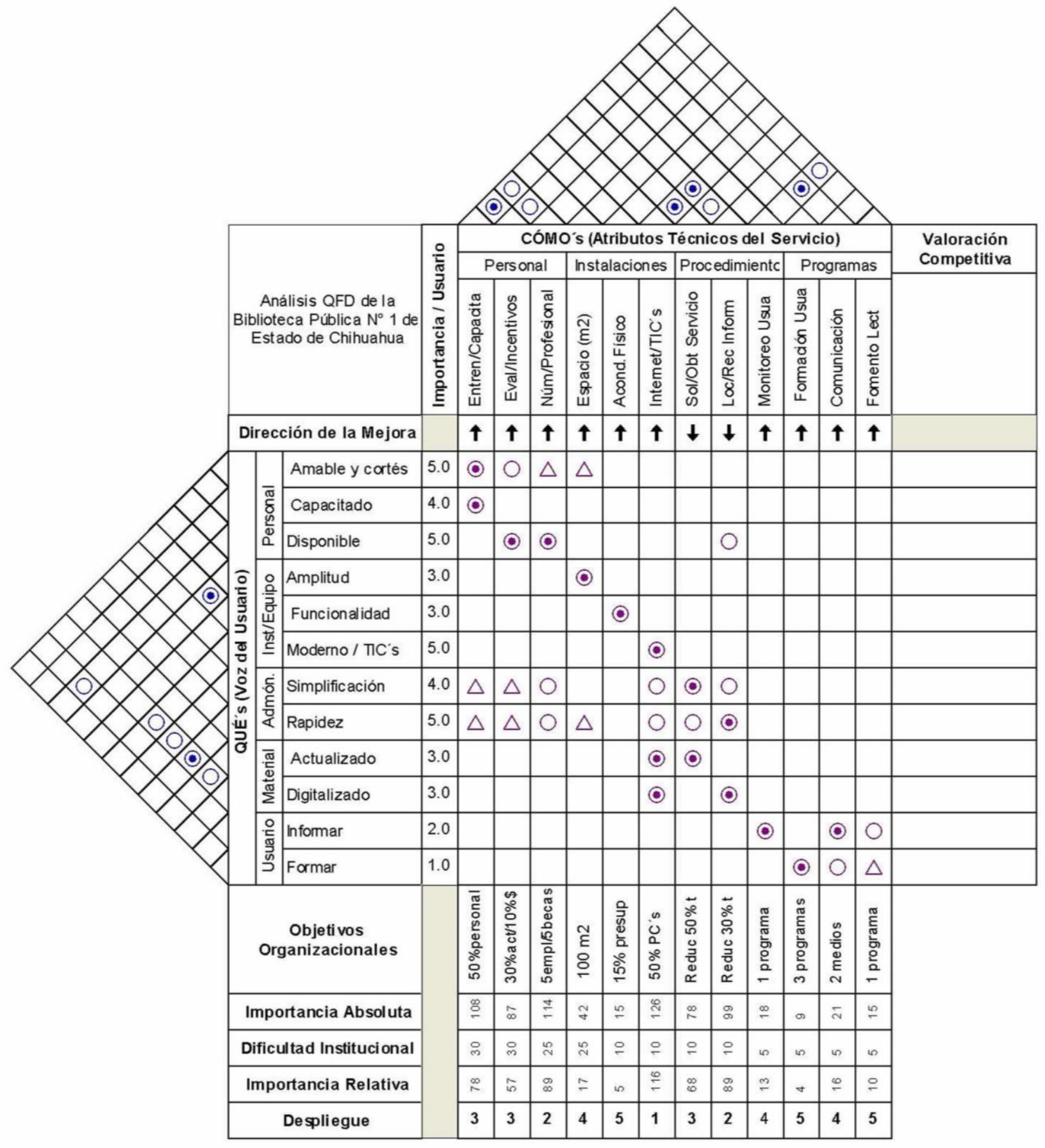

Fuente: Elaboración propia. 
La valoración competitiva es realizada por los usuarios al calificar los diversos aspectos en distintas bibliotecas consideradas como "competidoras", lo cual en este caso no es aplicable y por ello la matriz de la calidad se muestra vacía en este apartado. Como se puede observar en la casa de la calidad, las directrices estratégicas que se recomienda desplegar en orden jerárquico, en la biblioteca bajo análisis incluido en el Cuadro 5.

\section{Cuadro 5}

\section{Directrices estratégicas}

\begin{tabular}{|c|c|c|}
\hline $\begin{array}{c}\text { Factor } \\
\text { Organizacional }\end{array}$ & $\begin{array}{c}\text { Directriz } \\
\text { Estratégica }\end{array}$ & Posibles Acciones Estratégicas \\
\hline $\begin{array}{l}\text { Instalaciones y } \\
\text { equipamiento }\end{array}$ & $\begin{array}{l}\text { Mejorar el ambiente de trabajo de } \\
\text { manera que permita niveles } \\
\text { óptimos de estudio, realización de } \\
\text { tareas, trabajos en equipo y } \\
\text { actividades de esparcimiento, } \\
\text { procurando la automatización de } \\
\text { procesos y la digitalización de } \\
\text { servicios mediante la acentuación } \\
\text { en las nuevas TIC. }\end{array}$ & $\begin{array}{l}\text { Realizar un estudio integral de } \\
\text { los espacios físicos de la } \\
\text { biblioteca, con el apoyo de } \\
\text { especialistas, que busque la } \\
\text { adecuación ideal de acuerdo } \\
\text { con las funciones asignadas, } \\
\text { bajo un ambiente de } \\
\text { comodidad, iluminación } \\
\text { adecuada, bajo nivel de ruido, } \\
\text { buena ventilación y temperatura } \\
\text { ideal. } \\
\text { - Programa de modernización } \\
\text { tecnológica basado en TIC. }\end{array}$ \\
\hline $\begin{array}{c}\text { Personal de la } \\
\text { biblioteca }\end{array}$ & $\begin{array}{l}\text { Propiciar un ambiente de trabajo en } \\
\text { el cual el personal de la biblioteca } \\
\text { se sienta comprometido a } \\
\text { responder positivamente a las } \\
\text { demandas de los usuarios, } \\
\text { desarrollando sus capacidades y } \\
\text { aptitudes personales y } \\
\text { profesionales. }\end{array}$ & $\begin{array}{l}\text { Establecer un programa de } \\
\text { capacitación basado en las } \\
\text { cualidades del personal y } \\
\text { asignarles tareas donde } \\
\text { desarrollen sus habilidades y su } \\
\text { creatividad. } \\
\text { - Implantar un sistema de } \\
\text { evaluación e incentivos al } \\
\text { personal en función de la } \\
\text { atención al usuario. } \\
\text { - Profesionalizar en } \\
\text { bibliotecología al personal y/o }\end{array}$ \\
\hline
\end{tabular}




\begin{tabular}{|c|c|c|}
\hline $\begin{array}{c}\text { Factor } \\
\text { Organizacional }\end{array}$ & $\begin{array}{c}\text { Directriz } \\
\text { Estratégica }\end{array}$ & Posibles Acciones Estratégicas \\
\hline & & $\begin{array}{l}\text { contratar personal del área, } \\
\text { incluyendo becarios. } \\
\text { Analizar cargas de trabajo, } \\
\text { promover rotación de puestos y } \\
\text { formación de equipos de } \\
\text { trabajo. }\end{array}$ \\
\hline $\begin{array}{c}\text { Administración } \\
\text { bibliotecaria }\end{array}$ & $\begin{array}{l}\text { Mejorar el sistema administrativo, } \\
\text { enfatizando en la comunicación y } \\
\text { coordinación entre todos los } \\
\text { niveles, buscando su simplificación } \\
\text { y orientación al usuario. }\end{array}$ & $\begin{array}{l}\text { Revisar trámites para solicitar y } \\
\text { obtener servicios bibliotecarios } \\
\text { y simplificarlos al máximo para } \\
\text { brindar servicios más agiles y } \\
\text { eficientes al usuario. }\end{array}$ \\
\hline $\begin{array}{c}\text { Servicios } \\
\text { bibliotecarios }\end{array}$ & $\begin{array}{l}\text { Identificar, anticipar, proveer y } \\
\text { promover los servicios que } \\
\text { requieran los usuarios, de la forma } \\
\text { más efectiva y con conocimientos } \\
\text { especializados. }\end{array}$ & $\begin{array}{l}\text { Monitoreo constante de las } \\
\text { necesidades y requerimientos } \\
\text { de los usuarios, adecuando e } \\
\text { innovando los servicios en } \\
\text { función de la satisfacción del } \\
\text { usuario. }\end{array}$ \\
\hline $\begin{array}{c}\text { Materiales y } \\
\text { contenidos }\end{array}$ & $\begin{array}{l}\text { Actualizar materiales y contenidos } \\
\text { en función de la demanda de los } \\
\text { usuarios. }\end{array}$ & $\begin{array}{l}\text { Estudio diagnóstico de } \\
\text { materiales y contenidos, que } \\
\text { permita su actualización y } \\
\text { modernización ligada a la } \\
\text { demanda de los usuarios. }\end{array}$ \\
\hline Usuarios & $\begin{array}{l}\text { Formar a los usuarios de la } \\
\text { biblioteca en la búsqueda y } \\
\text { recuperación de información, } \\
\text { usando la gama completa de } \\
\text { servicios ofertados. }\end{array}$ & $\begin{array}{l}\text { - Programa de difusión de todos } \\
\text { los servicios que se ofrecen. } \\
\text { - Programas de formación de } \\
\text { usuarios. } \\
\text { - Programa de fomento a la } \\
\text { lectura. }\end{array}$ \\
\hline
\end{tabular}

Fuente: Elaboración propia

\section{Conclusiones}

Los análisis estratégicos de una biblioteca deben realizarse desde un enfoque sistémico, que permita considerar todos los elementos y subsistemas que afectan la marcha organizacional y no como ejercicios aislados que, aunque valiosos, pierden su utilidad al estar desalineados con 
respecto a otros análisis importantes tales como la gestión de la calidad de los servicios bibliotecarios y la medición del desempeño a través de ciertos indicadores, por mencionar las aplicaciones principales.

El sistema integral administrativo (SIAD) que se propone en la primera parte de este trabajo, es una propuesta sistémica que sustentada en un modelo de calidad, permite realizar la planeación estratégica de una biblioteca siguiendo las tres fases básicas de la administración estratégica, empleando también el cuadro de mando integral que permite implantar y darle seguimiento al desempeño de las estrategias de desarrollo tomando en cuenta cuatro perspectivas de las que sobresale la perspectiva del usuario, todo ello a través de los indicadores de desempeño ideados para monitorear los resultados organizacionales y retroalimentar todo el sistema. La propuesta deI SIAD se acompaña también con un ciclo de diseño, control, mejora e innovación (Ciclo DINOME) que permitiría alcanzar los objetivos institucionales de una manera armónica y sistematizada.

En lo referente a la aplicación del análisis QFD para escuchar y llevar la "voz del usuario" a cuestiones estratégicas para impulsar el desarrollo de una biblioteca, se concluye que este procedimiento administrativo es una herramienta útil y bondadosa que permite traducir los requerimientos del usuario en atributos técnicos que deben poseer los servicios bibliotecarios.

En definitiva, escuchar la voz del usuario para diseñar y desarrollar los servicios de una biblioteca e inclusive para realizar sus análisis estratégicos, se convierte hoy en día en una cuestión no solo de ventaja competitiva, sino de sobrevivencia institucional. Las bibliotecas deben salir de su encapsulamiento y escuchar las necesidades y requerimientos de los usuarios y analizar todas las nuevas tendencias en el uso y manejo de la información que traen aparejadas las características de esta nueva sociedad, llamada con justeza, "la sociedad de la información y el conocimiento".

El análisis QFD para las bibliotecas públicas del municipio de Chihuahua se basó en el estudio estadístico de usuarios a través de una encuesta, por lo que se recomienda explorar otros 
métodos cuantitativos y cualitativos para escuchar la voz de los usuarios, tales como el estudio de grupos focales, entrevistas segmentadas, buzón de quejas, etc., y llevarlos al QFD, tal como se hizo en el caso de este estudio.

\section{Referencias}

Aponte, R. y Chávez, H. (junio 2008). Proceso de acreditación de bibliotecas. Biblios, 31, 1-14. Bawden, D., Petuchovaite, R., Vilar, P. (2005). Are we effective? How would we know? Approaches to the evaluation of library services in Lithuania, Slovenia and the United Kingdom. New Library World, 106(9-10), 454-463.

Blázquez, M. y Medino, J. (octubre 2005). Atención al usuario en bibliotecas. Ponencia presentada en VII Encuentro de Bibliotecarios Municipales de Gran Canaria. Recuperado de http://eprints.rclis.org/8464/1/Encuentro_Sta_Brigida_Atencion_al_usuario.pdf

Borbely, M. (2011). Factors influencing self-assessment in a changing library environment. Performance Measurement and Metrics, 12(1), 7-22.

Boyce, C. (2017). Measuring perceptual (in)congruence between information service providers and users. College and Research Libraries, 78(3), 359-381.

Buck, W. (2016). Organizational integration, strategic planning, and staff assessment in publicly funded libraries. Public Services Quarterly, 12(4), 277-289.

Chin, K.; Pun, K.; Leung, W. y Lau, H. (2001). A Quality Function Deployment approach for Improving Technical Library and Information Services: A Case Study. Library Management, 22(1), 195-204.

Dabbour, K. y Kott, K. (2017). Dialogic approaches to strategic planning in academic libraries: an appreciative inquiry case study at Oviatt Library. Journal of Library Administration, 257(4), 468-480.

David, F. (2003). Conceptos de administración estratégica. (9a Edición). México, D.F.: Pearson Educación - Prentice Hall.

De Melo, Á. y De Alencar, I. (2016). Theoretical framework of performance indicators with BSC for the private higher education institution. IFIP Advances in Information and Communication Technology, 488, 452-458. 
Evans, J. R., Lindsay, W. (2005). Administración y control de la calidad. (5ª Edición). México, D.F.: International Thomson Editores.

Friss, I. (2003). Modelo para la Creación de Entornos de Aprendizaje basados en técnicas de Gestión del Conocimiento. (Tesis doctoral) Doctor en Ingeniería Informática, Universidad Politécnica de Madrid, España.

Fuentes, J. (2001). Planificación de centros bibliotecarios. Revista General de Información y Documentación, 11(1), 49-82.

Fuentes, J. (2002). La planificación estratégica aplicada a las bibliotecas nacionales: la encuesta de Nueva Zelanda. Boletín de la Asociación Andaluza de Bibliotecarios, 68, 921.

García, J. (2009). Análisis y propuesta metodológica del Despliegue de la Función de Calidad en Procesos de una biblioteca universitaria. (Tesis doctoral) Universidad Nacional de Educación a Distancia, Escuela Técnica Superior de Ingenieros Industriales, España.

Gatti, M. (2008). Diseño e implementación del módulo de ingresos para el Sistema Administrativo Integrado Descentralizado (SAID). (Tesis) Universidad de Los Andes, Argentina.

González, O. (mayo-agosto 2012). La dirección estratégica en las bibliotecas universitarias. Ciencias de la Información, 43(2), 15 - 20.

Gutiérrez, H.; Gutiérrez, P.; Garibay, C. y Díaz, L. (2014). Análisis multivariado y QFD como herramientas para escuchar la voz del cliente y mejorar la calidad del servicio. Ingeniare: Revista Chilena de Ingeniería, 22(1), 62-73.

Herrera-Viedma, S.; López, J. y Vílchez, J. (2006). Un modelo de evaluación de la calidad en las bibliotecas universitarias digitales basado en técnicas difusas. Enc. Bibli: R. Eletr. Bibliotecon. Ci. Inf., Florianópolis, Número especial, $2^{\circ}$ semester.

Hunt, R. y Xavier, F. (2003). The leading edge in strategic QFD. International Journal of Quality and Reliability Management, 20(1), 56-73.

Kaplan, R. y Norton, D. (1996). The balanced scorecard. Boston, Mass.: Harvard Business School Press. 
Kundanis, B. (2014). Succession planning and the library: The strategic plan. En Springs, E. (Ed.). Succession planning and implementation in libraries: Practices and resources (pp. 180-201). Hershey, Estados Unidos: IGI Global.

Madrid, I. (2002). Evaluación de Bibliotecas: su necesidad e importancia. Información, Cultura y Sociedad, 6, 103-113.

Martín-Montalvo, I. (2007). Estudio de satisfacción de usuarios en la red de bibliotecas del Instituto Cervantes. Revista General de Información y Documentación, 17(2), 9-30.

Melo, B. y Zavala, E. (2012). Propuesta de un sistema de indicadores de desempeño para la acreditación de un centro de información de una institución de educación superior. (Tesis de Maestría en Administración con Especialidad en Alta Dirección) Instituto de Estudios Superiores de Tamaulipas, México.

Ministerio de Sanidad y Consumo (España). (2007). Marco Estratégico para la mejora de la Atención Primaria en España: 2007-2012. Madrid, España: El Ministerio.

Moreno, J. (2007). Guía para la aplicación del modelo EFQM en entidades de acción social: cuaderno de gestión 2. Madrid, España: Fundación Juan Vives.

Omella, E.; Permanyer, J. y Vilagrosa, E. (desembre 2009). Instrumentos básicos para la planificación estratégica del servicio de biblioteca pública. BID: Textos Universitarios de Biblioteconomía y Documentación, 23, 1-13.

Pacios, A. (2017). Public library planning: a routine practice? Library Management, 38(4-5), 237247.

Robledillo, A. y Velázquez, D. (2013). Introducción a los Sistemas de Gestión de la Calidad Total: Modelo de Excelencia EFQM y Autoevaluación. Medicina y Seguridad del Trabajo, 59(232), 302-309.

Secretaría de Educación Pública. (2005). Modelo de innovación y calidad. Oficialía Mayor. Dirección General de Innovación, Calidad y Organización. México, D.F.: SEP.

Tamayo, F. y González, V. (2009). ¿Qué es el QFD? Descifrando el despliegue de la función de calidad. Asociación Latinoamericana de QFD. Recuperado de http://www.qfdlat.com/Imagenes/QFD.pdf

Universidad Autónoma de Chihuahua, Facultad de Filosofía y Letras. (2011). Plan de desarrollo de la Facultad de Filosofía y Letras 2011-2021. Chihuahua, México: UACH. 
Varela, P. (2012). Aplicación del despliegue de la función de calidad (QFD) para la evaluación y mejoramiento del producto Openenglish.com. (Tesis de Especialista en Generación de Proyectos) Universidad Católica Andrés Bello, Caracas, Venezuela.

Villardefrancos, M. (2004). La orientación al cliente en las organizaciones de información. Recuperado de http://www.bibliociencias.cu/gsdl/collect/eventos/index/assoc/HASH018b.dir/doc.pdf Vonhof, C. y Bertele, M. (2017). "Excellent Library" - A Holistic Quality Model and Certificate for Libraries: Concept and Experiences from Germany. International Information and Library Review, 49(1), 19-30.

\section{Notas de los Autores}

José Refugio Romo-González: Correo electrónico: iromo@uach.mx; ORCID: http://orcid.org/0000-0002-4810-4357, Universidad Autónoma de Chihuahua, México.

Javier Tarango: Correo electrónico: jtarango@uach.mx; ORCID: http://orcid.org/0000-00020416-3400; Universidad Autónoma de Chihuahua, México

Juan D. Machin-Mastromatteo: Correo electrónico: imachin@uach.mx, ORCID: http://orcid.org/0000-0003-4884-0474, Universidad Autónoma de Chihuahua, México. 